\title{
PID Control of Heat Exchanger System
}

\author{
Yuvraj Bhushan Khare \\ Yaduvir Singh \\ Department of Electrical \& Instrumentation Engineering
Thapar University, Patiala, 147001, Punjab, India
}

\begin{abstract}
Heat exchanger system is widely used in chemical plants because it can sustain wide range of temperature and pressure. The main purpose of a heat exchanger system is to transfer heat from a hot fluid to a cooler fluid, so temperature control of outlet fluid is of prime importance. To control the temperature of outlet fluid of the heat exchanger system a conventional PID controller can be used. Due to inherent disadvantages of conventional control techniques, model based control technique is employed and an internal model based PID controller is developed to control the temperature of outlet fluid of the heat exchanger system. The designed controller regulates the temperature of the outgoing fluid to a desired set point in the shortest possible time irrespective of load and process disturbances, equipment saturation and nonlinearity. The developed internal model based PID controller has demonstrated 84\% improvement in the overshoot and $44.6 \%$ improvement in settling time as compared to the classical controller.
\end{abstract}

\section{Keywords}

Feed-forward controller, internal model based PID controller, PID Controller and heat exchanger.

\section{INTRODUCTION}

In practice, all chemical process involves production or absorption of energy in the form of heat. Heat exchanger is commonly used in a chemical process to transfer heat from the hot fluid through a solid wall to a cooler fluid. There are different types of heat exchanger used in the industry but most of the industry used heat exchanger system [11]. Heat exchangers are probably the most common types of heat exchanger applicable for wide range of operating temperature and pressure. They have larger ration of heat transfer surface to volume than double pipe heat exchangers, and they are easy to manufacture in large variety of sizes and configuration. They can operate at high pressure, and their construction facilitates disassembly for periodic maintenance and cleaning. Heat exchanger find widespread used in refrigeration, power generation, heating and air-conditioning, chemical process, manufacturing, and medical application. A heat exchanger in an extension of the double pipe configuration. Instead of single pipe within a larger pipe, a heat exchanger consists of bundles of pipes or tubes enclosed within a cylindrical shell. In the heat exchanger one fluid flows through a tubes and a second fluid flows through within the space between the tubes and the shell.

This paper reports a work that considers a heat exchanger and builds a single-input single outputs model of the system with the help of experimental data available. The outlet temperature of the heat exchanger system has to be kept at a desired set point according to a process requirement. Firstly a classical PID controller is implemented in a feedback control loop so as to achieve the control objectives. PID controllers exhibits high overshoot which is undesirable. To reduce the overshoot and optimize the control performance, a feed forward controller is used along with a feedback controller. The combined effect of feedback and feed forward control schemes gives a much better result than the feedback PID controller.

\section{HEAT EXCHANGER SYSTEM}

A typical interacting chemical process for heating consists of a chemical reactors and a heat exchanger system. The process fluid which is the output of the chemical reactor is stored in the storage tank. The storage tank supplies the fluid to the heat exchanger system. The heat exchanger heats up the fluid to a desired set point using super heated steam at $180 \mathrm{C}$ supplied from the boiler. The storage tank supplies the process fluid to a heat exchanger system using a pump and a non returning valve. The super heated steam comes from the boiler and flows through the tubes, whereas the process fluids flow through the shells of the heat exchanger system. After the steams heat up the process fluid, the condensed steam at $100 \mathrm{C}$ goes out of the heat exchanger system. There is also a path of non condensed steam to go out of the shell and heat exchanger system in order to avoid the blocking of the heat exchanger.

Different assumptions have been considered in this research paper. The first assumption is that the inflow and the outflow rate of fluid are same, so that the fluid level is maintained constant in the heat exchanger. The second assumption is the heat storage capacity of the insulating wall is negligible. In this feedback process control loop, the controller is reverse acting, the valve used is of air to open (fail-close) type. A thermocouple is used as the sensing element, which is implemented in the feedback path of the control architecture. The temperature of the outgoing fluid is measured by the thermocouple and the output of the thermocouple (voltage) is sent to the transmitter unit, which eventually converts the thermocouple output to a standardized signal in the range of 4-20 ma. This output of the transmitter unit is given to the controller unit. The controller implements the control algorithm, compares the output with the set point and then gives necessary command to the final control element via the actuator unit. The actuator unit is a current to pressure converter and the final control unit is an air to open (fail-close) valve. The actuator unit takes the controller output in the range of 4-20 ma and converts it in to a standardized pressure signal, i.e. in the range of 3-15 psig. The valve actuates according to the controller decisions. Figure 1 show the basic feedback control scheme implemented in a heat exchanger system.

There can be two types of disturbances in this process, one is the flow variation of input fluid and the second is the temperature variation of input fluid. But in practice the flow variation of input fluid is a more prominent disturbance 
than the temperature variation in input fluid. So, in feed forward control loop, the input fluid flow is measured and the disturbance in the flow is controlled using a feed forward controller. The output of the feedback and the feed forward controller is added and the resultant output is given to the control valve. With the addition of feed forward controller the control performance is further optimized.

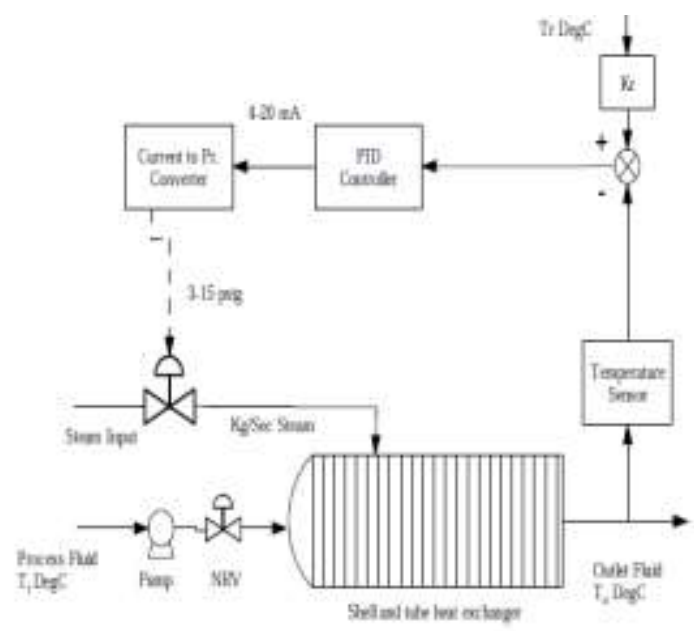

Figure 1: Heat Exchanger System Control Scheme

\section{MATHEMATICAL MODELLING}

Figure 2 shows conventional control block diagram consisting of process, controller, actuator, final control element, sensor, set point and different kind of process disturbances.

From the experimental data, the transfer functions and gains are obtained as below:

$$
\text { Transfer function of process } \frac{50 e^{-s T_{d}}}{30 s+1}
$$

Gain of valve

0.13

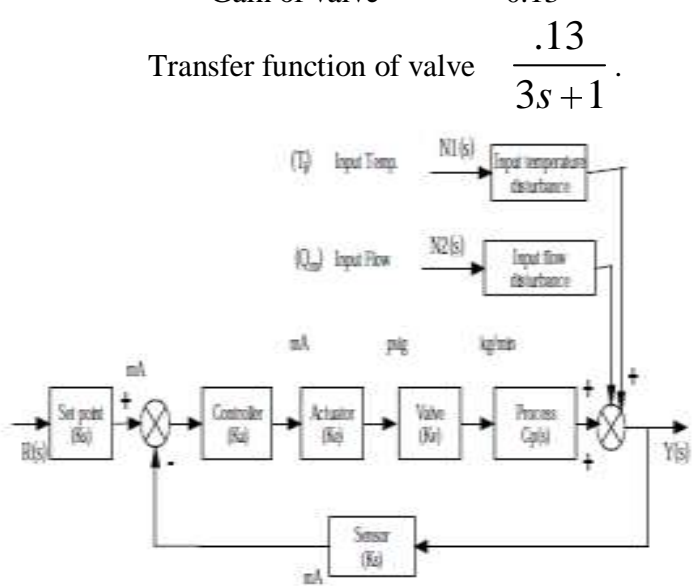

Figure 2: Block diagram for feedback control of heat exchanger system.

Gain of I/P converter $\quad 0.75$

Transfer function of disturbance variables (flow and temperature disturbance respectively)

$$
\frac{1}{30 s+1}, \frac{3}{30 s+1}
$$

Transfer function of thermocouple $\quad \frac{0.16}{10 s+1}$

To control the exit temperature of the heat exchanger system a classical feedback controller is used. The PID control algorithm remains the most popular approach for industrial process control despite continual advances in control theory. This is not only due to the simple structure which is conceptually easy to understand and, which makes manual tuning possible, but also to the fact that the algorithm provides adequate performance in the vast majority of applications.

The characteristic equation $(1+\mathrm{G}(\mathrm{s}) \mathrm{H}(\mathrm{s})=0)$ in this case is obtained as below.

$900 s^{3}+420 s^{2}+43 s+0.798 k_{c}+1=0$

Routh stability criterion gives $k_{c}$ as 23.8

Auxiliary Equation $420 s^{2}+0.798 k_{c}+1=0$

$\omega=0.218$ and $\mathrm{T}=28.79$

PID controller in continuous time is given as

$$
u(t)=k_{c}\left(e(\bar{t})+\frac{1}{\tau_{i}} \int_{0}^{t} e(t) d t+\tau_{d} \frac{d e(t)}{d t}\right)
$$

The transfer function of PID controller is

$$
\begin{aligned}
& G_{\text {pid }}(s)=\frac{u(s)}{e(s)}=k_{c}\left(\frac{\tau_{i} \tau_{d} s^{2}+\tau_{i} s+1}{\tau_{i} s}\right) \\
& G_{p i d}(s)=\frac{u(s)}{e(s)}=k_{c}\left(\frac{\tau_{i} s+1}{\tau_{i} s}\right)\left(\frac{\tau_{d} s+1}{1+\tau_{f} s}\right) \\
& G_{p i d}(s)=\frac{u(s)}{e(s)}=k_{c}\left(\frac{\tau_{i} s+1}{\tau_{i} s}\right)\left(\frac{\tau_{d} s+1}{1+\alpha \tau_{f} s}\right)
\end{aligned}
$$

Most of the PID tuning rules developed in the last sixty years use frequency-response methods [6,8]. Examples include, Ziegler-Nichols rule, symmetric optimum rule, Ziegler-Nichols' complementary rule, some-overshoot rule, no-overshoot rule, refined Ziegler-Nichols rule, integral of squared time weighted error rule, and integral of time absolute error rule [7]. These methods are straightforward to apply since they provide simple tuning formulae to determine the PID controller parameters. However, since only a small amount of information on the dynamic behaviour of the process is used, in many situations they do not provide good enough tuning or produce a satisfactory closed-loop response. In practice, the Ziegler-Nichols rule often leads to a rather oscillatory response to set point changes. From its invention in 1940s Zeigler-Nichols tuning rule is used extensively in industries to tune the PID controllers.

According to Zeigler-Nichols frequency response tuning criteria $\mathrm{K} \mathrm{p} \square 0.6 \mathrm{Kc}, \square \mathrm{i} \square 0.5 \mathrm{~T}$ and $\square \mathrm{d} \square 0.125 \mathrm{~T}$

For the PID controller in the heat exchanger, the values of tuning parameters obtained are $\mathrm{Kp}=14.28, \tau \mathrm{i}=14.395$, $\tau \mathrm{d}=3.59$ and $\mathrm{P}=23.8, \mathrm{I}=1.65, \mathrm{D}=85.442$

Usually, initial design values of PID controller obtained by all means needs to be adjusted repeatedly through computer simulations until the closed loop system performs or compromises as desired. In order to have a good closedloop time response, the following performance functions are considered during the design of a PID controller. 


$$
\begin{aligned}
& J\left(K_{p}, K_{i}, K_{d}\right)=\int_{0}^{\infty}(r(t)-y(t))^{2} d t \\
& J\left(K_{p}, K_{i}, K_{d}\right)=\int_{0}^{\infty} t|(r(t)-y(t))| d t
\end{aligned}
$$

Thus, the optimal PID controller design is stated as min $\mathrm{J}$ $(\mathrm{K} \mathrm{p}, \mathrm{K} \mathrm{i}, \mathrm{Kd})$

The disturbance input introduces error in the system performance. In several systems the disturbance can be predicted and its effect can be eliminated with the help of feed forward controller before it can change the output of the System.

In feedback control scheme the sensor detects the process output and gives the error to the controller, which in turn takes appropriate controlling action. But till the controlling action reaches the process, the output has been changed. So a feed forward controller along with the feedback controller has been implemented. A feed forward control estimates the error and changes the manipulating variable before the disturbance can affect the output. To further minimize the overshoot a feed-forward controller is introduced in the forward path of the process along with the feedback controller. The combined effect of feedback and feedforward controller reduces the overshoot value. Figure 4 shows the transfer function representation of system with feedback and feed-forward controller.

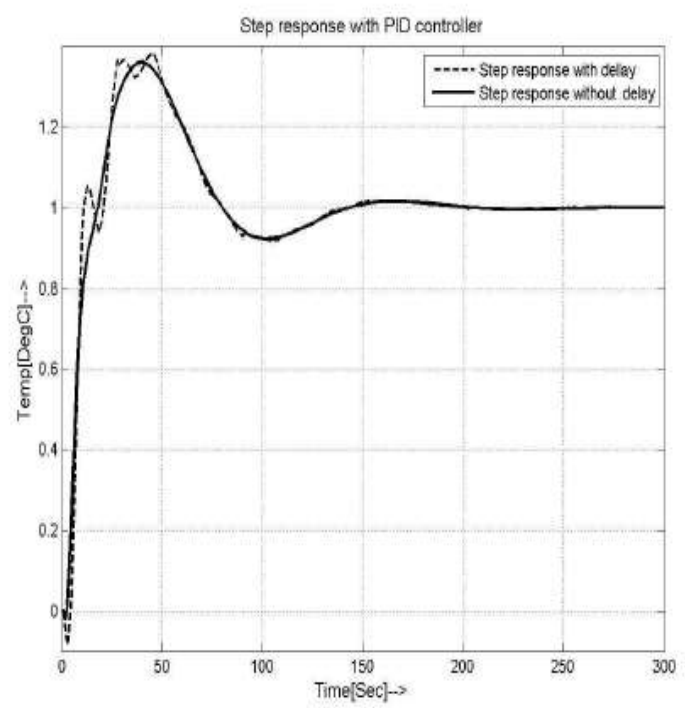

Step response of heat exchanger system with feedback controller.

$$
G_{p}(s)=\frac{5 e^{-s T}}{90 s^{2}+33 s+1}, G_{d}(s)=\frac{1}{30 s+1}
$$

The transfer function of the feed-forward controller is

$$
\begin{aligned}
& G_{c f}(s)=\frac{-G_{d}(s)}{G_{p}(s)} \\
& G_{c f}(s)=\frac{-18 s^{2}-6.6 s-0.2}{30 s+1 \lambda s+1}
\end{aligned}
$$

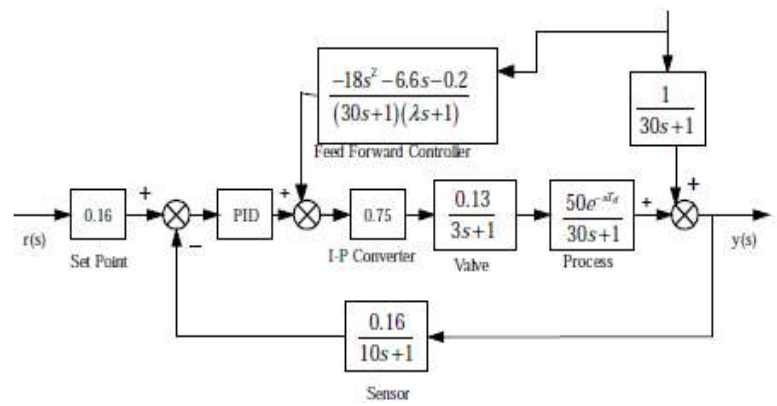

Figure 4: Feed forward and feedback control block diagram of heat exchanger system.

\section{INTERNAL MODEL BASED PID CONTROLLER}

The most common industrial controller is PID controller and internal model control scheme shown in figure 7 can be used to design a standard feedback PID controller. The standard feedback controller is a function of the internal model and internal model controller $\mathrm{Q}(\mathrm{s})$. The transfer function of feedback controller equivalent to internal model controller is shown in eq. (12).

$$
G_{c}(s)=\frac{Q(s)}{1-\tilde{G}_{p}(s) Q(s)} .
$$

Substituting the values in eq. (12) the transfer function of the feedback controller based on internal model controller is obtained as shown in eq. (13).

$$
G_{c}(s)=\frac{90 s^{2}+33 s+1}{5\left(\lambda^{2} s^{2}+2 \lambda s\right)} .
$$

Equating the terms of eq. (5) and eq. (13), the values of PID parameters are obtained.

PID controller for improved disturbance rejection can be achieved by changing the internal model controller filter. For improved disturbance rejection the filter is in the form of

$$
\mathrm{F}(\mathrm{s})=\frac{\gamma s+1}{(\lambda s+1)^{n}}
$$

Here $\gamma$ is selected to achieve good disturbance rejection. It is selected to cancel slow disturbance time constant. Several methods of tuning of internal model controller and efficient calculation of filter parameter have been proposed in literature [1].

\section{SIMULATION AND TESTING}

The simulations for the different control mechanism discussed above were carried out in Simulink and the simulation results have been obtained. Figure 5 represents the Simulink modelling of the heat exchanger system with PID as a feedback controller. 


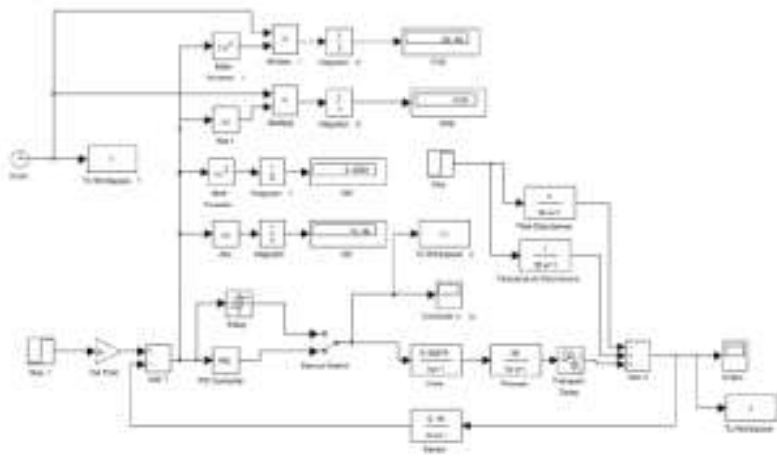

Figure 5: Simulink model of heat exchanger system with feedback PID controller.

As shown in figure 5 for auto-tuning of PID controller, a relay block is connected in parallel with the PID controller with the help of a manual switch. When the auto-tune function is required, the manual switch is set to the relay block. The relay block represents a nonlinear behaviour. In auto-tune mode, the closed loop system oscillates and the manipulated variable action is ON-OFF. From the auto-tune mode, two parameters are obtained. These parameters are ultimate gain and ultimate frequency. Ultimate gain and ultimate frequency are given as

Ultimate gain

$$
K_{c u}=\frac{4 h}{\pi a}
$$

Ultimate frequency

$$
\omega_{u}=\frac{2 \pi}{p}
$$

Here $P$ is the period between the successive peaks a is the amplitude of process output

\section{$\mathrm{h}$ is the height of controller output}

The behaviour obtained from auto tuning mode is very similar to the behaviour obtained from Zeigler- Nichols closed loop cycling method.

Figure 6 shows the step response of the heat exchanger system with PID controller with and without transportation delay.

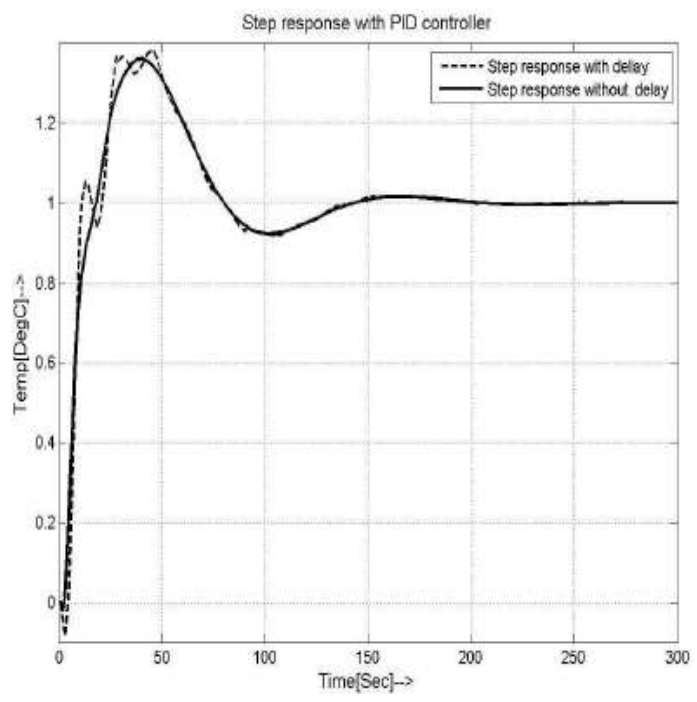

\section{Step response of heat exchanger system} with feedback controller.

Figure 7 shows the Simulink model of heat exchanger system with both feedback and feed-forward controller. The advantage of feed-forward control strategy is that a disturbance variable is measured and a manipulated variable is changed before the output is affected. But the main disadvantage of this kind of control strategy is sensitivity to uncertainty. If the inlet flow rate is not perfectly measured or the steam input can't be manipulated perfectly, then the outlet temperature of heat exchanger will not be perfectly controlled. With any small disturbances the output temperature can't be controlled in an effective manner.

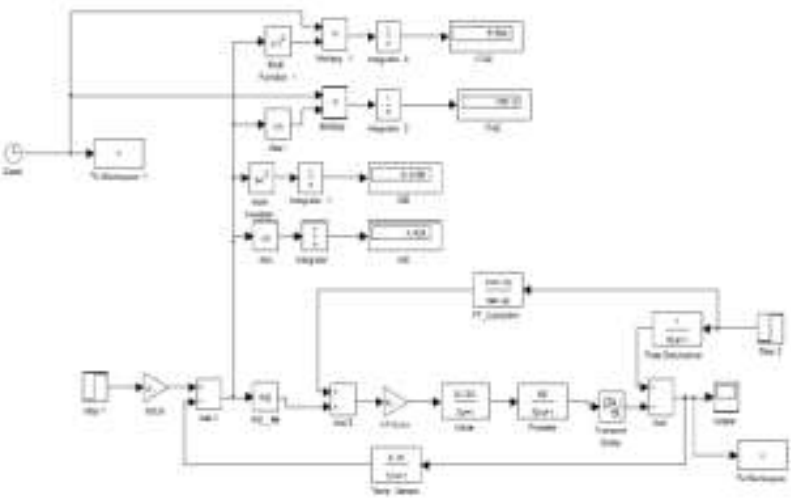

Figure 7: Simulink model of heat exchanger system with feedback and feed forward controller.

To implement a feed-forward control strategy prior knowledge of the process disturbances and mathematical model of the process must be known to the process engineer.

Figure 8 shows the step response of the heat exchanger system with feedback and feed forward controller. The combined effect of feedback and feed forward controller reduces the overshoot and also decreases the settling time by $21 \%$.

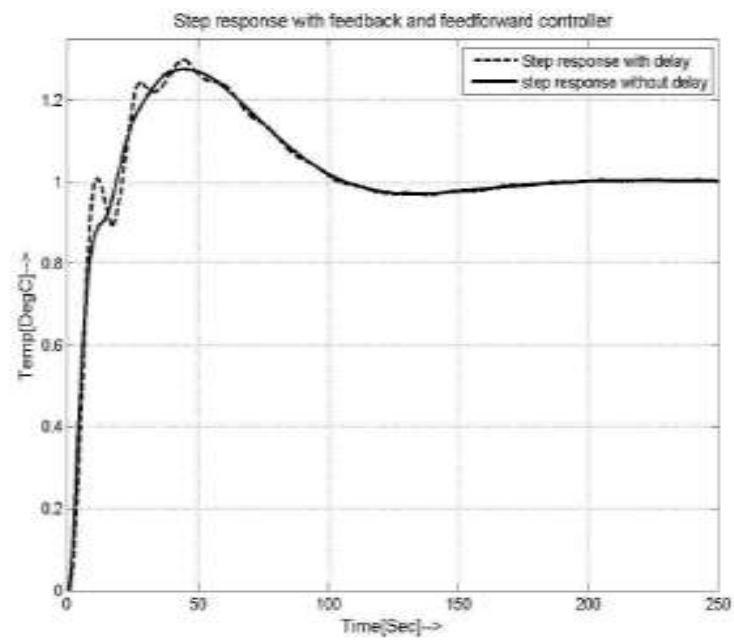

Figure 8: Step response of heat exchanger system with feedback and feed forward controller.

Figure 9 shows the Simulink model of heat exchanger system with internal model based PID controller. Internal model based PID controller carries a significant advantage over conventional PID controller as there is only one tuning parameter where as there are three tuning parameter in 
conventional PID controller.

Figure 10 shows the step response of heat exchanger system when an internal model based PID controller is used to control the controlling variable. The maximum overshoot is only $5 \%$ and the controller meets both steady state and transient state criteria.

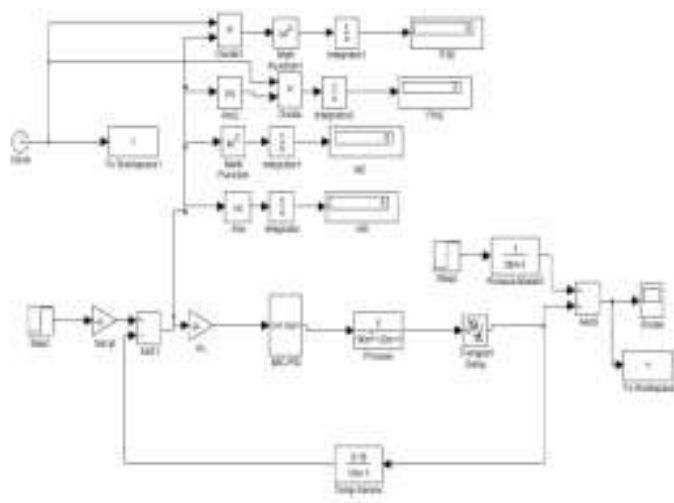

Figure 9: Simulink model of internal model based PID controller.

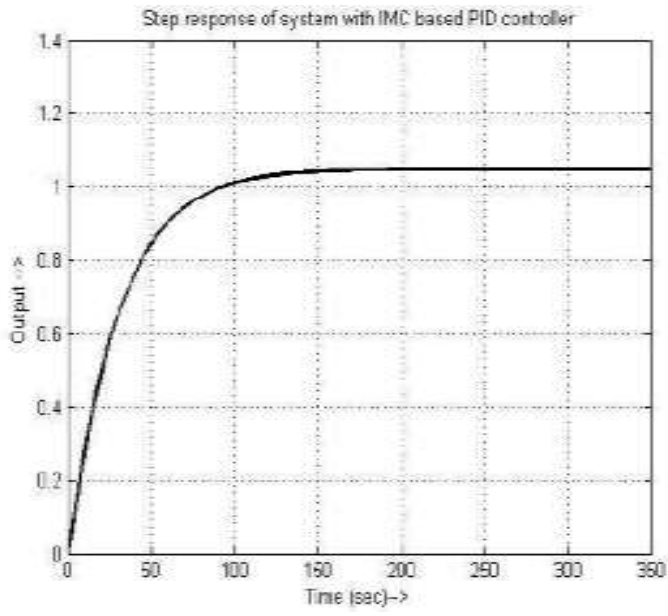

Figure 10: Step response of internal model based PID controller.

To evaluate the performance of the different controllers this paper has considered two vital parameters of the step response of the system. The first parameter is the maximum overshoot and the second parameter is the settling time.

\section{RESULT AND DISCUSSION}

In the time domain, specifications for a control system design involve certain requirements associated with the time response of the system. The requirements are often expressed in terms of the standard quantities on the rise time, settling time, overshoot, peak time, and steady state error of a step response. In all the four controllers these two parameters are evaluated. A comparative study of their performance has been in the table 1 below.
TABLE I

COMPARISION OF DIFFERTRNT PARAMETERS EN CONTROLIFS

\begin{tabular}{|l|l|l|l|}
\hline S.No & & $\begin{array}{l}\text { Overshoot } \\
(\%)\end{array}$ & $\begin{array}{l}\text { Settling } \\
\text { Time (sec) }\end{array}$ \\
\hline 1 & Feedback PID & 38.38 & 115.2 \\
\hline 2 & $\begin{array}{l}\text { Feedback plus feed- } \\
\text { forward controller }\end{array}$ & 30.04 & 91.3 \\
\hline 3 & Internal model controller & 16 & 77.9 \\
\hline 4 & $\begin{array}{l}\text { Internal model based } \\
\text { PID controller }\end{array}$ & 5 & 63.8 \\
\hline
\end{tabular}

From the above observations it is clear that in conventional PID controller in feedback loop the heat exchanger produces an overshoot is $38.38 \%$. To compensate this kind of high overshoot a feed forward controller in conjunction with the conventional PID in feedback loop is implemented. By implementing this method the system overshoot was reduced to $30 \%$, an improvement of $21 \%$. Though the overshoot has some what decreased, it can be further decreased by implementing internal model based controller and internal model based PID controller. By implementing internal model based PID controller the overshoot reduces to $5 \%$ respectively. In feedback controller the settling time was $115.2 \mathrm{sec}$ where as in feed forward plus feedback controller the settling time decreases to $91.34 \mathrm{sec}$, an improvement of $20.7 \%$. By implementing internal model based PID controller the settling time decreases by $63.8 \mathrm{sec}$. From these observations it is clear that internal model based PID controller is a much better option for control rather than conventional feedback and feedback plus feed forward controller.

\section{CONCLUSIONS}

This paper takes a case study of heat exchanger system and evaluates different methods to control the outlet fluid temperature. Four different kinds of controllers are designed to control the outlet temperature of fluid and the performances of these controllers are evaluated by two different methods. One of the methods for performance evaluation is the time domain analysis of overshoot and settling time and other method is calculation of performance Indices. Firstly a classical PID controller is designed to achieve the control objective. But due to the unsatisfactory performance of the PID controller a feed forward controller is designed and placed in the forward path of the system. To further increase the efficiency of the system the internal model based PID controller is designed and implemented. The internal model based PID controller gives satisfactory performance in both steady state and transient state in time domain analysis. The performance indices of all the controllers are also evaluated.

This paper takes the process model to be the same as the process, which is practically impossible to achieve. So as a further work we can implement direct model and inverse model based controller and apply system identification as well as neural networks concepts for estimation of process model. 


\section{REFERENCES}

[1] Fernando G. Martins, "Tuning PID Controllers Us ing the ITAE Criterion," International Journal of Engineering Education, vol. 21, no. 3, 2005.

[2] G.K.I. Mann, B. G. Hu and R.G Gosine, "TimeDomain Based Design and Analysis of New PID Tuning Rules," IEEE Proceedings of Control Theory Application, vol. 148, no. 3, , 2001, pp. 251-261

[3] G.P. Liu, S. Daley, "Optimal Tuning PID Control for Industrial Systems," Control Engineering Practice, 9, 2001, pp. 1185-1194.

[4] Ian G Horn et.al, "Improved Filter Design in In ternal Model Control," Industrial Engineering Chemistry Research, 35, 1996, pp. 3437-3441 .

[5] Ibrahim Kaya and Nusret Tan, "Controller Design for Stable Processes Using User Specified Gain and Phase Margin Specifications and Two Degreeof-Freedom IMC Structure," in Proceedings of 11th Mediterranean Conference on Control and Automation, 2003

[6] Kiam Heong Ang, Gregory Chong and Yun Li, "PID Control System Analysis, Design, and Technology," IEEE transaction on control system technology, vol. 13 , no. 4, 2005, pp. 559-576

[7] Myungsoo Jun and Michael G. Sofonov, "Automatic PID Tuning: An Application of Unfalsified Control," IEEE Symposium on CACSD, 1999, pp. 1-5.
[8] Orlando Duran et.al, "Neural Networks for Cost Estimation of Shell and Tube Heat Exchangers," in Proceedings of International Multi Conference of Engineers and Computer Scientists, vol II, 2008.

[9] R. Vilanova et.al, "A Framework for Disturbanc e Attenuation on SISO Stable/Unstable Systems", in Proceedings of $7^{\text {th }}$ World Congress on Intelligent Control and Automation, 2008, pp. 2976-29.

[10] Satean Tunyasrirut et.al, "The Auto-Tuning PID Controller for Interacting Water Level Process," in Proceedings of World Academy of Science, Engineering and Technology, vol.12, 2006, pp. 134-138.

[11] T N Luan et.al, "Design of Multi-Loop PID Controllers Based on the generalized IMC-PID Method with Mp Criterion," International Journal of Control, Automation, and Systems, vol. 5, no. 2, 2007, pp. 212-217.

[12] Wen Tan et.al, "Comparison of Some well-known PID Tuning Formulas," Computers and Chemical Engineering, 30, 2006, pp. 1416-1423. 